\title{
Assessing Clinicians' Reliance on Computational Aids for Acute Stroke Diagnosis
}

\author{
Vishwajith Ramesh \\ Dept. of Bioengineering \\ University of California, San Diego \\ La Jolla, CA 92093, USA \\ vramesh@eng.ucsd.edu \\ Brett C. Meyer \\ Stroke Center \\ University of California, San Diego \\ La Jolla, CA 92093, USA \\ bcmeyer@health.ucsd.edu
}

\author{
Andrew Nguyen \\ Dept. of Cognitive Science \\ University of California, San Diego \\ La Jolla, CA 92093, USA \\ andrewdnguyen64@gmail.com \\ Gert Cauwenberghs \\ Dept. of Bioengineering \\ University of California, San Diego \\ La Jolla, CA 92093, USA \\ gert@ucsd.edu
}

\author{
Kunal Agrawal \\ Stroke Center \\ University of California, San Diego \\ La Jolla, CA 92093, USA \\ kuagrawal@health.ucsd.edu \\ Nadir Weibel \\ Dept. of Computer Science \\ University of California, San Diego \\ La Jolla, CA 92093, USA \\ weibel@ucsd.edu
}

\begin{abstract}
The rapid rise of computational aids for stroke diagnosis have led to important concerns about clinicians developing an overdependence on technology. Other studies have assessed reliance on clinical decision support systems in fields like diabetes, but no such study exists for stroke diagnosis. In this work, we developed a highfidelity user interface for a computational aid designed to support acute ischemic stroke diagnosis. Engaging with stroke practitioners at the UCSD Stroke Center, we conducted an experiment to determine how technology for identifying stroke symptoms may affect their diagnostic decision-making processes. By assessing how clinicians changed their video-based diagnosis of stroke when provided with data visualizations and predictions from a machine learning tool, we observed that such computational aids do in fact affect clinicians' decisions but only in cases when the aid directly supports or contradicts their prior beliefs. Future computational aids for stroke diagnosis should focus on helping clinicians solidify their decisions rather than only providing them with overly quantitative information that may impede or confuse their judgement.
\end{abstract}

\section{KEYWORDS}

Stroke Diagnosis, Clinician Reliance, Ubiquitous Computing, Computational Aids, Machine Learning, Clinical Decision Support, Automation Bias

\section{ACM Reference Format:}

Vishwajith Ramesh, Andrew Nguyen, Kunal Agrawal, Brett C. Meyer, Gert Cauwenberghs, and Nadir Weibel. 2020. Assessing Clinicians' Reliance on Computational Aids for Acute Stroke Diagnosis. In 14th EAI International Conference on Pervasive Computing Technologies for Healthcare (PervasiveHealth '20), May 18-20, 2020, Atlanta, GA, USA. ACM, New York, NY, USA, 10 pages. https://doi.org/10.1145/3421937.3422019

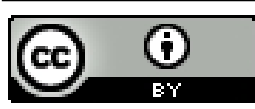

This work is licensed under a Creative Commons Attribution International 4.0 License. PervasiveHealth '20, May 18-20, 2020, Atlanta, GA, USA

(C) 2020 Copyright held by the owner/author(s).

ACM ISBN 978-1-4503-7532-0/20/05.

https://doi.org/10.1145/3421937.3422019

\section{INTRODUCTION}

Acute stroke diagnosis presents several challenges that motivate the development and use of computational aids. Stroke diagnosis typically requires a neurological examination done by a clinician in a hospital - the National Institute of Health Stroke Scale (NIHSS) [17, 20]. The NIHSS is a set of motor and cognitive tests designed to quickly and quantitatively assess impairments. It is a widely adopted exam that has been shown to be useful for rehabilitation planning. However, it is not a perfect test for diagnosis. Follow-up imaging revealed that $21 \%$ of patients treated for stroke based on the results of the NIHSS did not in fact have strokeassociated brain infarcts [8]. In addition, while useful for identifying large vessel occlusions, the NIHSS cannot properly identify stroke mimics, false positive stroke cases that represent up to $30 \%$ of acute stroke hospital admissions [2, 31]. (Stroke mimics are non-vascular conditions like brain tumors or migraine that present with similar symptoms as acute ischemic stroke.) With a specificity of only $52 \%$, the NIHSS cannot properly filter out stroke mimics, often resulting in unnecessary diagnostic procedures that waste neurologists' and patients' time [2]. Quickly diagnosing stroke is important because ideally, acute ischemic stroke is diagnosed within 3 hours from the presentation of symptoms. Tissue plasminogen activator (tPA), the only FDA-approved therapy for ischemic stroke, must be administered in that time for it to be effective [3]. The increasing demand for immediate and accurate diagnoses has led to the emergence of computational approaches to aid stroke evaluation [23-26, 34].

Computational diagnostic aids quicken the recognition of stroke and help reduce errors by providing decision support in a limited amount of time [19]. Several researchers have developed machine and deep learning-based systems for stroke diagnosis to support the NIHSS and improve clinical outcomes [5, 22, 35]. Recent work capitalizes on advances in machine learning and ubiquitous technology (namely wearables and depth cameras that provide body pose) to computationally identify and reliably quantify the degree of different stroke-related deficits [23, 24, 26].

\subsection{Over-Dependence on Technological Aids}

When deciding to adopt digital healthcare technology, hospitals and clinics often prioritize cost and ability to improve workplace 
efficiency. However, the risk that that technology may build blind trust and negatively impact the decision making process of clinicians is often ignored [27, 29, 33]. In fact, over-dependence on technology was discovered to be an unintended adverse consequence of clinical information systems; Campbell et al. and Ash et al. both found that clinicians could not work efficiently without computerized systems and that they had false expectations about data accuracy and processing $[4,7]$. It was this latter issue that concerned us about artificial intelligence (AI) diagnostic tools. Various diagnostic AI techniques have been proven to be capable of solving numerous clinical problems yet clinicians are hesitant to include them in their decision making processes [13]. It is important to balance computational approaches for diagnosing stroke with clinicians' assessments, to augment them rather than replace them [29]. This balance is necessary to promote the adoption of AI tools in healthcare environments.

Our neurologist collaborators were concerned that practitioners outside the field of neurology like EMS and less experienced stroke specialists like residents may become over-dependent on technological aids for stroke diagnosis, ignoring their own assessments. In fact, in the area of psychotherapy, Hirsch et al. observed that novice counselors generally accepted the accuracy of what was presented to them by a machine learning-based automated evaluation and assessment system [12]. Motivated by this concern, we desired a better understanding of clinician reliance on software designed to improve decision making in acute stroke diagnosis. To do so, we took a similar approach as a study by Sims et al. in the area of diabetes care [30]. Sims et al. developed and tested a new type of interface for a diabetes-specific clinical decision support system that displayed laboratory results in a color-coded way for the quick understanding of diabetics' metabolic control [30]. They evaluated the effects of their dashboard on the identification of patients who required adjustments in their treatment. Doctors performed timed mock clinical tasks with and without the dashboard and rated their confidence in interpreting diabetes-related test results. By uncovering the effects of their dashboard on clinicians' decisions for adjusting treatment, Sims et al. learned best practices for integrating their support system into existing workflows [30]. No such study yet exists for AI-based computational aids for acute stroke diagnosis.

In this paper, we describe the development of an initial user interface (UI) for a stroke diagnostic tool based on prior work and feedback from a focus group of 10 stroke clinicians. We designed and conducted an online experiment in which we evaluated whether computational aids influenced an otherwise standard, video-based stroke diagnosis. We discovered that clinicians seemed to consider computational aids only when the aids confirmed or denied their loosely held beliefs.

\section{METHODS}

In order to understand how computational aids affected stroke specialists' decision making, we conducted a focus group at the UCSD Stroke Center. The results of the focus group informed the design of a prototype UI for an acute stroke diagnosis computational aid that we then evaluated in an experimental setting.

\subsection{Stroke Center Focus Group}

The focus group consisted of 10 clinicians working at the UCSD Stroke Center, including registered nurses, medical residents, and experienced neurologists. Participants were asked to: (1) outline concerns about the introduction of AI-based diagnostic technology into their clinic, (2) identify gaps in the information typically available to them, and (3) list additional information that would be valuable for acute diagnosis.

The focus group revealed that a tool that supports clinicians during stroke evaluation needs to quickly convey information. Focus group participants also expressed the desire to see quantitative data about the stroke symptoms identified by a computational system. For example, they wanted to be able to see graphs that could be used to understand how the system detected certain stroke symptoms. Finally, while the prospect of a computational aid was received positively, clinicians worried about the use of such a tool by less experienced clinicians. Specifically, they wondered how less experienced stroke practitioners would balance their own assessments with the predictions of a computational aid, especially in cases of disagreements between the two.

\subsection{Designing a User Interface}

Based on feedback from the focus group and prior work done in the setting of user interfaces for stroke diagnosis, we designed the initial prototype UI shown in Figure 1.

We detail two key visual features of the UI relevant to this study to measure clinician reliance (additional details can be found in Ramesh et al. "Developing Aids to Assist Acute Stroke Diagnosis" [25]):

(1) Skeleton of Deficits: In earlier work, Gotoh et al. designed a figure with different body parts highlighted to represent the symptoms of a stroke patient [11]. We used a similar representation and created a skeleton image to easily visualize different deficits of the body parts, shown in Figure 1A. An important benefit of using the skeleton was that our system used 3D spatial coordinates of human body joints (obtained with depth cameras such as the Microsoft Kinect or from video with deep learning methods for human pose estimation) to identify symptoms like hemiparesis [24, 26]. A skeleton visual enabled us to place a clickable node on each body joint to plot a joint's X, Y, or Z position in space over time.

(2) Plotting Joint Position: Several stroke studies plot the movement and velocity of body joints in the upper extremities and pupil position (obtained via video-oculography) over time $[16,18]$. Inspired by this approach and the fact that the NIHSS is comprised primarily of motor tests that assess symptoms ranging from weakness to tremors, we presented quantitative position information of key joints to clinicians using graphs like the one in Figure 1B. The $\mathrm{X}$ axis represented time and the $\mathrm{Y}$ axis represented the movement of a body joint in $\mathrm{X}$, Y, or Z coordinate space. Since our machine learning system for symptom identification relied on 3D spatial coordinates as input, the graph was included to help the user better understand how an AI-based computational aid arrived at a prediction $[24,26]$. 


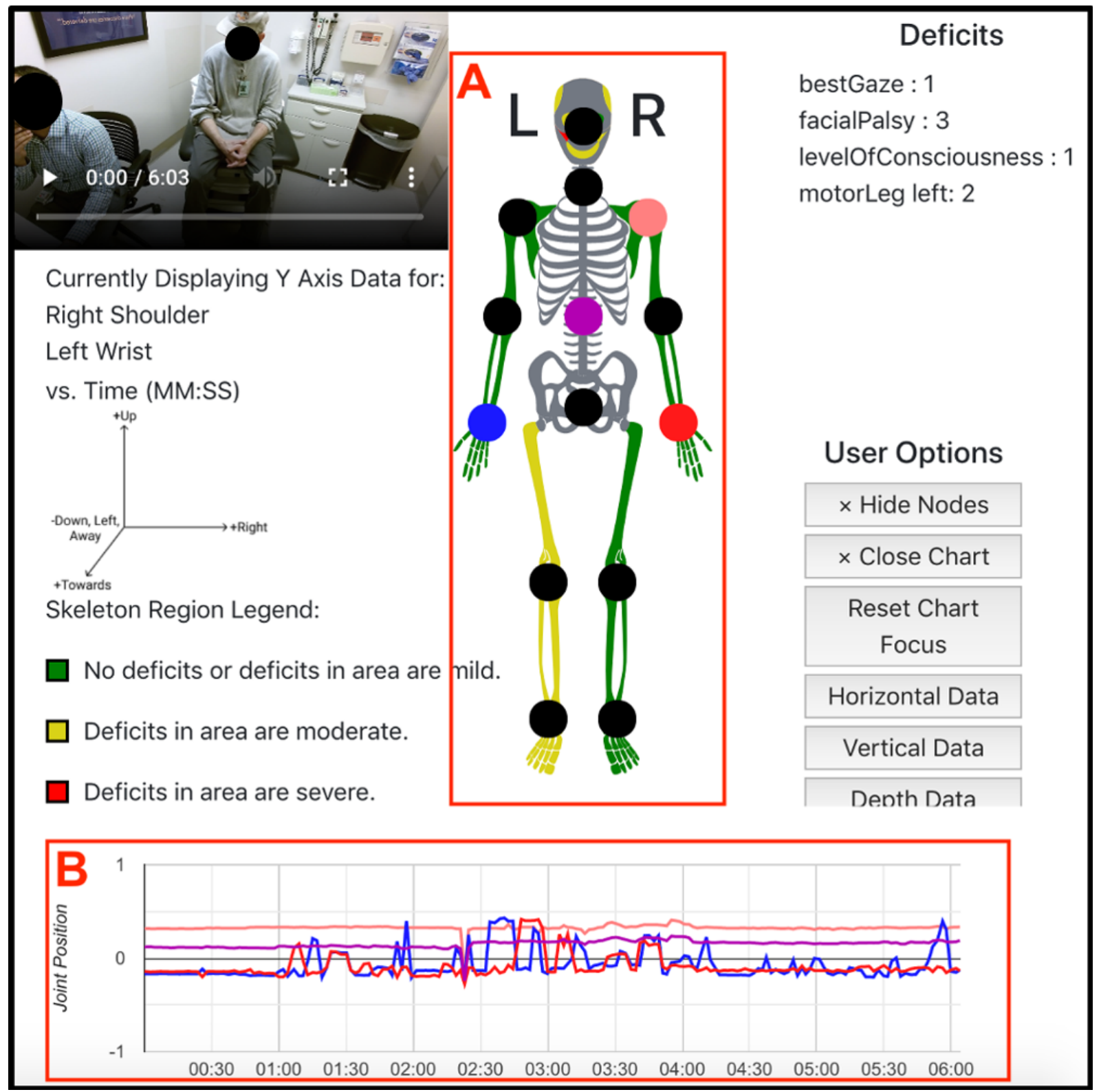

Figure 1: Initial user interface prototype of a clinical decision support system for acute stroke diagnosis evaluated in this study. High-definition video recording (with audio) of a stroke patient undergoing an in-person NIHSS was displayed on the top left. Deficits identified by the system were listed in the top right. (A) A 3-color skeleton showed symptoms and their severities (red for most severe, yellow for moderate symptoms, and green for no deficits). Black, clickable nodes represented the joints for which we could display $3 \mathrm{D}$ spatial position data. (B) The user could choose whether to have a joint's horizontal $\mathrm{X}$ position (left/right), vertical Y position (up/down), or depth $\mathrm{Z}$ position (away/towards) displayed in a graph over time. The horizontal position data of the left wrist (blue), right wrist (red), core (purple), and right shoulder (peach) over time are plotted.

We created a high-fidelity dynamic web interface using HTML, CSS, and React.js. Users were able to watch and listen to recordings of stroke patients undergoing an NIHSS exam and to view plots of the spatial positions of body joints over the duration of the inperson NIHSS exam. The graphs and the video were synced (as in [10]). So users could jump to any timestamp in the video and be shown the body joint spatial positions at that timestamp, and vice-versa.

We wanted to better understand clinician reliance on technological aids in stroke diagnosis to inform the development of a computational pipeline. (An understanding of the user would give us guidelines for the design of a final interface.) Since a real-time, 


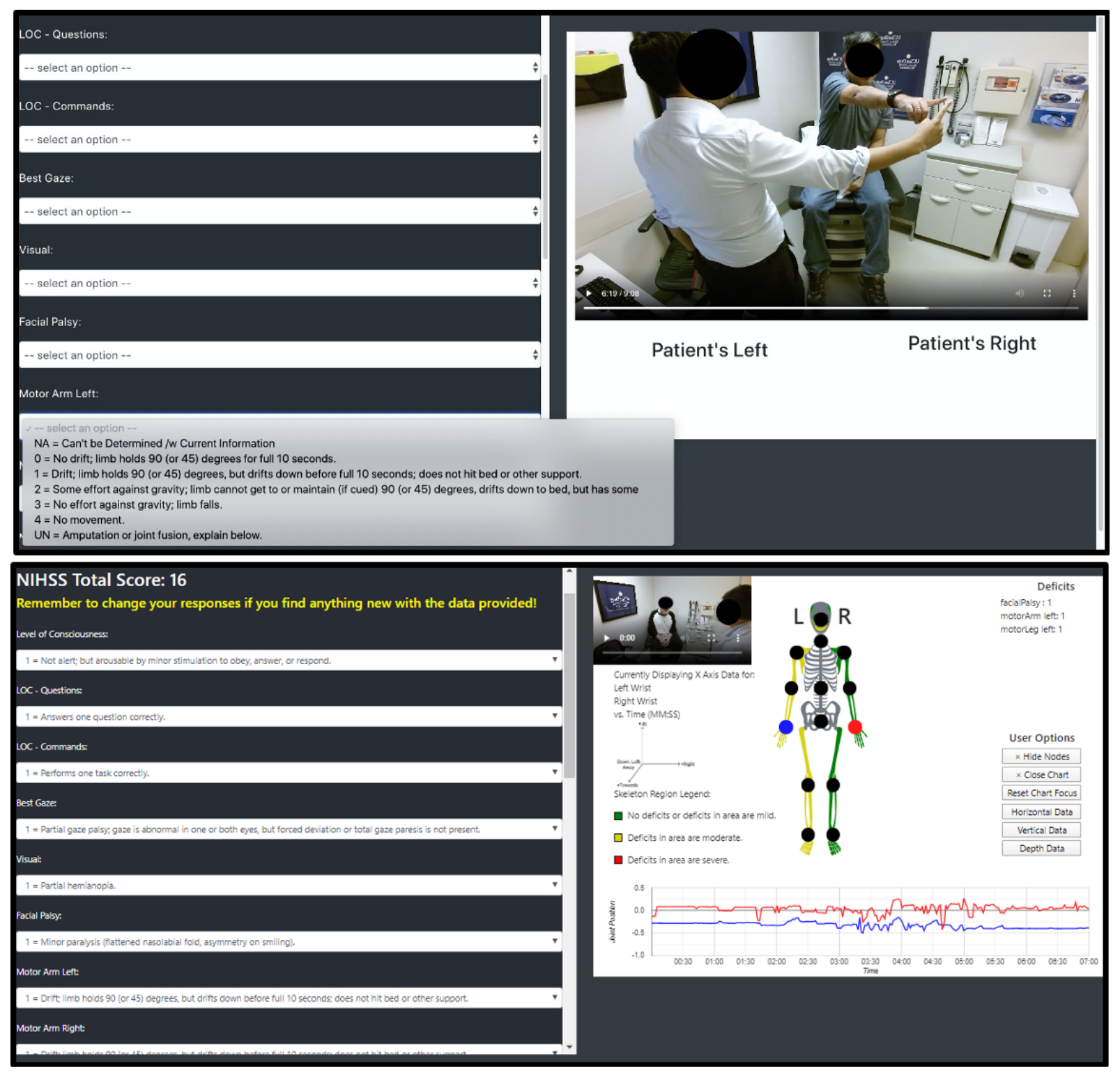

Figure 2: In the first part of the experiment (top), participants watched video footage (with audio) of a neurologist conducting the NIHSS in-person and filled out a drop-down menu version of the NIHSS. In the second part (bottom), participants had the option to change their responses based on what was presented to them in the UI, detailed in Figure 1.

fully functional system was still under development at the time of the experiment, we filled the UI prototype with previously recorded data from stroke patients. We recorded 8 stroke patients while a neurologist was conducting the NIHSS at UCSD Stroke Center outpatient clinics. We followed the same data collection protocol outlined in [24]. We used the Microsoft Kinect v2 depth camera to record audio, video, and the 3D position of 25 body joints over time. For each of the 8 patients, we obtained the neurologist's diagnosis, a list of symptoms identified, and their associated NIHSS scores. After obtaining the NIHSS results for the 8 patients, we displayed the recorded video, the identified symptoms and their severity, and the recorded body joint position information in the UI prototype as shown in Figure 1. All patients agreed to be recorded with sensors by signing a consent form approved by the local Human Research Protections Program office.

We categorically represented a stroke deficit's severity with a 3 -color scale to enable quick identification of symptoms. Each of the tests in the NIHSS exam has a different scoring system, with some deficits scored out of 3 and others out of 2 or 4 [20]. To implement a consistent 3-color scale for all symptoms, we designed the skeleton to have 11 components or areas: left/right eyes, left/right face, left/right arm, left/right leg, left/right head, and mouth. The NIHSS 
test scores associated with each area were summed. For example, the NIHSS tests related to the right leg measured ataxia of the right leg, sensory loss in the right leg, and drift of the right leg. If all 3 NIHSS scores were 0 , the right leg on the skeleton was colored green. If at least one of the 3 scores was 1 , the area was colored yellow. Red was used to indicate the highest severity, when the total was greater than or equal to the number of related NIHSS scores. In the case of the motor leg, a total $>=3$ would be colored red. We conducted this analysis for all 11 defined areas on the skeleton.

\subsection{Assessing Clinicians' Reliance}

To determine whether a clinical decision support system for stroke diagnosis would influence the decision making of clinicians, we designed an experiment and survey to evaluate our prototype UI. The experiment was designed to take 1.5 to 2 hours to complete and could be done in parts to accommodate the busy schedules of clinician participants.

2.3.1 Overview of Experiment Structure. In stroke telemedicine, stroke specialists can diagnose a potential stroke case occurring in another location by "calling in" via video conferencing software. Typically, specialists coordinate with a nurse or member of emergency medicine staff onsite who does an in-person NIHSS. Stroke diagnosis via telemedicine has been shown to be about as effective as diagnosis with an in-person NIHSS, especially when the remote stroke specialist has access to brain imaging [1]. Video-based stroke assessments have similarly been shown to be useful even without brain imaging available [28]. Therefore, in our experiment, we asked participants to watch the recorded footage (video and audio) of stroke patients undergoing an in-person NIHSS and to diagnose stroke to the best of their ability.

Participants were presented with 7 cases (not including 1 patient used as a tutorial to acclimate participants to the experiment's interface). Each case consisted of 2 parts. In the first, participants filled out a drop-down form of the NIHSS exam while using the recorded footage to identify symptoms and diagnose stroke (see Figure 2). Participants rated their confidence in their video-based assessments from 1 (least confident) to 7 (most confident). They also had the option to include general notes and feedback, especially if they felt that they could not properly assess a symptom from video and audio. (This approach was inspired by a similar healthcare study related to spirometry interpretation that rated the perceived confidence of participants out of 7 [21]. Studies in non-healthcare fields (relating to answering trivia questions or eyewitness identifications, for example) have also used a 7 point scale for confidence $[6,14]$.)

In the second part of each case, participants were shown the prototype UI from Figure 1 that listed the deficits identified by the neurologist conducting the in-person NIHSS. Participants' NIHSS scores and confidence ratings from the first part (the left side of Figure 2) were saved and carried over. In the second part, participants were given the option to change their answers from the first part in light of the new information provided to them through the UI. This information included the body joint position graphs and the identified deficits and their severities. Participants were told that this information was detected by the computational AI system, but the deficits and severities listed were actually determined by the stroke specialist conducting the in-person NIHSS in the video.
Table 1: Clinical Roles of Experiment Participants

\begin{tabular}{|c|c|}
\hline Participant & Clinician Role \\
\hline $\mathbf{1}$ & Stroke Fellow \\
\hline $\mathbf{2}$ & Neurologist \\
\hline $\mathbf{3}$ & Neurologist \\
\hline $\mathbf{4}$ & Nurse Practitioner \\
\hline $\mathbf{5}$ & Stroke Fellow \\
\hline $\mathbf{7}$ & Stroke Fellow \\
\hline
\end{tabular}

To assess reliance on computational aids, we monitored changes made in the second part of the experiment. Participants were again asked to rate their confidence in their NIHSS diagnoses after using the computational aid. They were also asked if they felt that any of the displayed symptoms were incorrect, to which they had the option to respond "Yes" or "No".

After completing the tutorial and all 7 cases, we presented participants with a final, post-experiment survey that included questions regarding: (1) their confidence in the accuracy of the information that was shown by the UI, (2) whether they had difficulty diagnosing any symptoms with the information provided, (3) what sources of information they generally trusted and regularly used in their decision making, and (4) which UI elements they found to be most and least helpful.

2.3.2 Recruiting Participants. Our study was conducted in collaboration with the UCSD Stroke Center. To recruit participants, we sent a link to the online experiment and survey to the center, comprised of neurologists, fellows, residents, nurses, and nurse practitioners. Note that none of the experiment's participants were the neurologist conducting the NIHSS in person. The participants of the experiment were not involved in the diagnosis or rehabilitation of any of the patients used in the experiment.

We collected responses from a total of 6 clinicians who used our online prototype. Out of the 6, 4 also finished the post-experiment survey. Table 1 shows the roles of the participants: we had responses from 3 stroke fellows, 1 nurse practitioner, and 2 full-time, more experienced, neurologists.

2.3.3 Manipulating the Ground Truth. The in-person neurologist's NIHSS assessments were the basis of real-life treatment and rehabilitation planning for the 8 recorded stroke patients. As such, we considered the in-person scores as "ground truth". In particular, we used the in-person NIHSS results in the UI prototype as a proxy to our computational AI-based system (still in development at the time of this study).

To properly measure dependence on the computational aid, we gauged how clinicians responded to discrepancies between their own assessments and the results of the system shown on the UI. (After all, the final, fully developed system may still make erroneous predictions far from the ground truth.) In each case and in the final, post-experiment survey, participants were asked whether they believed the information shown on the prototype UI was accurate. We slightly manipulated the in-person NIHSS results shown to participants in the UI to identify if participants caught the deliberately introduced deviations from the ground truth. 3 out of the 7 stroke patient cases were randomly chosen and manipulated. In the first 
Table 2: Actual Symptoms and NIHSS vs. Values Presented*

\begin{tabular}{|c|c|c|c|c|}
\hline \multirow{2}{*}{ Pt. } & \multicolumn{2}{|c|}{ Ground Truth } & \multicolumn{2}{|c|}{ Values Shown in UI } \\
\hline & Symptoms & NIHSS & Symptoms & NIHSS \\
\hline \multirow{2}{*}{1} & Motor Arm Left & 1 & Motor Arm Left & 1 \\
\hline & Motor Leg Left & 1 & Motor Leg Left & 1 \\
\hline \multirow{3}{*}{2} & Facial Palsy & 1 & Facial Palsy & 1 \\
\hline & Motor Arm Left & 3 & Motor Arm Left & 1 \\
\hline & Motor Leg Left & 1 & Motor Leg Left & 1 \\
\hline \multirow{2}{*}{3} & Motor Arm Left & 1 & Motor Arm Left & 2 \\
\hline & Motor Leg Left & 1 & Motor Leg Left & 3 \\
\hline 4 & \multicolumn{2}{|c|}{ Healthy (No Symptoms) } & & \\
\hline 5 & \multicolumn{2}{|c|}{ Healthy (No Symptoms) } & & \\
\hline 6 & \multicolumn{2}{|c|}{ Healthy (No Symptoms) } & Motor Arm Right & 2 \\
\hline 7 & Visual & 2 & Visual & 2 \\
\hline
\end{tabular}

${ }^{*} \mathrm{Pt}=$ Patient, Manipulations highlighted

case, we slightly changed the NIHSS score of a random symptom identified by the in-person neurologist to another random number. In the second case, for each identified symptom, we changed the score to a random score. In the third case, we listed a deficit that was not identified by the in-person neurologist and so was not actually present in the stroke patient. See Table 2. Participants were given the ability to change their NIHSS assessments from the first part when these manipulated results were shown to them in the second part.

\section{RESULTS}

Results are summarized in Table 3 and Table 4 . Table 3 includes the symptoms presented by the 7 patients, as identified by a stroke specialist conducting an in-person NIHSS. It also lists the symptoms identified by experiment participants for each patient/case. Experiment participants identified symptoms after seeing video only (in the first part of each case) and were given the option to make changes to their assessment after being shown the prototype UI in Figure 1 (in the second part of each case). Table 3 lists symptoms (and associated severities) from both parts.

Table 4 focuses on whether participants made changes to their video-based assessments after seeing the prototype UI. For each patient/case, Table 4 notes whether participants made changes to either the symptoms they identified or the associated severities. Table 4 also lists the difference in reported confidence between the first part and the second part and whether participants thought the UI shown in the second part was incorrect.

\subsection{Participant Confidence in Video-Based NIHSS Assessments}

In the first part of the experiment, participants rated their confidence in their ability to diagnose stroke from videos of the NIHSS. The stroke fellows and experienced neurologists were generally confident in their video-based NIHSS assessments; scores were mainly 5 or higher out of 7 . Participant 4 , the nurse practitioner, was the least confident with scores of either $4 / 7$ or $5 / 7$.

\subsection{Changes Made After Seeing Prototype UI and Manipulations}

The NIHSS scores and symptoms of Patients 2, 3, and 6 were manipulated as shown in Table 2. For example, for Patient 2, we reduced the motor arm left NIHSS score from $3 / 4$ to $1 / 4$. For such cases with manipulated data, we analyzed how clinicians changed their scores, if they did. We looked for correlations between our manipulations and the changes the clinicians made to their self-reported confidence.

4 out of 6 participants made at least 1 change after seeing the UI in the second part of the experiment; only Participants 3 and 5 made no changes to their initial video-based assessments (see Table 4). For example, as can be seen in Table 3, Participant 1 identified a motor symptom in Patient 3's left leg only after seeing the UI. After seeing the UI, Participant 1 not only made note of this motor issue but also caught an error they made in the first part - the symptom they initially identified in the patient's right arm was switched to the left arm (Table 3). Note however that in the first part, Participant 1 identified several symptoms that Patient 3 did not present with (such as facial palsy and limb ataxia). Participant 1 kept these symptoms in the second part even though the UI did not have them listed (Table 2).

As with Participant 1, Participant 2 also caught an error after seeing the UI. Participant 2 initially and erroneously thought Patient 1 did not have motor symptoms but changed their assessment to incorporate the scores presented to them in the UI (Table 3). Interestingly, Participant 2 initially scored a deficit in Patient 5 that they then removed after seeing the UI, ultimately giving Patient 5 a healthy diagnosis, which was correct. For Patient 1, Participant 2's confidence decreased from 6/7 to 5/7, as noted in Table 4. For Patient 5, Participant 2's confidence increased from 5/7 to 6/7 (Table 4). Also consider Participant 4, who, after seeing the UI, removed a motor symptom they initially identified in Patient 4 (Table 3).

\subsection{Belief in the Accuracy of Displayed Results}

Table 4 shows the responses of participants when they were asked about the accuracy of the results shown on the prototype UI. Of particular interest are the manipulated patients - Patients 2, 3, and 6. While the results and data shown in the UI for (healthy) Patient 5 were deemed accurate by all of the participants, they unanimously distrusted the manipulated results shown for Patient 6 . The belief in the accuracy of the displayed results for Patients 2 and 3 were more mixed.

\subsection{Final Survey Results}

After the experiment was over, clinicians were asked to complete a short survey about the prototype interface and to answer specific UI-related questions. The survey revealed that despite not being in person, participants did not have difficulty diagnosing most stroke symptoms using video footage (with audio) and the prototype UI. (This was also evidenced by the strong confidence values reported earlier in Section 3.1.) Some participants had difficulty assessing facial palsy and best gaze symptoms due to lighting conditions in the video (reported as "N/A" values in Table 3). 1 participant reported a desynchronization of the video and audio due to technical issues. We believe this effect to be negligible as none of the patients 
Table 3: Symptoms Identified by Participants in First Part of Experiment (Video Only) and Second Part (with UI)*

\begin{tabular}{|c|c|c|c|c|c|c|c|c|c|c|c|c|c|c|}
\hline \multirow{3}{*}{ Pt. } & \multirow{2}{*}{\multicolumn{2}{|c|}{ In-Person Neurologist }} & \multicolumn{4}{|c|}{ Participant 1} & \multicolumn{4}{|c|}{ Participant 2} & \multicolumn{4}{|c|}{ Participant 3} \\
\hline & & & \multicolumn{2}{|c|}{ Part 1 (Video) } & \multicolumn{2}{|c|}{ Part 2 (UI) } & \multicolumn{2}{|c|}{ Part 1 (Video) } & \multicolumn{2}{|c|}{ Part 2 (UI) } & \multicolumn{2}{|c|}{ Part 1 (Video) } & \multicolumn{2}{|c|}{ Part 2 (UI) } \\
\hline & Symptoms & NIHSS & Symptoms & NIHSS & Symptoms & NIHSS & Symptoms & NIHSS & Symptoms & NIHSS & Symptoms & NIHSS & Symptoms & NIHSS \\
\hline \multirow{3}{*}{1} & Motor Arm Left & 1 & Visual & 1 & Visual & 1 & Best Gaze & $\mathrm{N} / \mathrm{A}^{* *}$ & Best Gaze & $\mathrm{N} / \mathrm{A}^{* *}$ & Visual & 1 & Visual & 1 \\
\hline & Motor Leg Left & 1 & Limb Ataxia & 1 & Limb Ataxia & 1 & & & Motor Arm Left & 1 & & & & \\
\hline & & & & & & & & & Motor Leg Left & 1 & & & & \\
\hline \multirow{6}{*}{2} & Facial Palsy & 1 & Dysarthria & 1 & Dysarthria & 1 & Dysarthria & 1 & Dysarthria & 1 & Dysarthria & 1 & Dysarthria & 1 \\
\hline & Motor Arm Left & 3 & Facial Palsy & 1 & Facial Palsy & 1 & Facial Palsy & 1 & Facial Palsy & 1 & Facial Palsy & 2 & Facial Palsy & 2 \\
\hline & Motor Leg Left & 1 & Motor Arm Left & 1 & Motor Arm Left & 1 & Motor Arm Left & 3 & Motor Arm Left & 3 & Motor Arm Left & 1 & Motor Arm Left & 1 \\
\hline & & & Motor Leg Left & 1 & Motor Leg Left & 1 & Motor Arm Right & 1 & Motor Arm Right & 1 & Motor Leg Left & 1 & Motor Leg Left & 1 \\
\hline & & & & & & & Motor Leg Left & 2 & Motor Leg Left & 2 & & & & \\
\hline & & & & & & & Motor Leg Right & 1 & Motor Leg Right & 1 & & & & \\
\hline & Motor Arm Left & 1 & Best Language & 1 & Best Language & 1 & Dysarthria & 1 & Dysarthria & 1 & LoC Questions & 1 & \begin{tabular}{|l} 
LoC Questions \\
\end{tabular} & 1 \\
\hline & Motor Leg Left & 1 & Facial Palsy & 1 & Facial Palsy & 1 & Facial Palsy & 1 & Facial Palsy & 1 & Motor Arm Left & 1 & Motor Arm Left & 1 \\
\hline & & & Limb Ataxia & 1 & Limb Ataxia & 1 & Limb Ataxia & 2 & Limb Ataxia & 2 & & & & \\
\hline 3 & & & LoC Questions & 1 & LoC Questions & 1 & Motor Arm Left & 1 & Motor Arm Left & 1 & & & & \\
\hline & & & Motor Arm Right & 1 & Motor Arm Left & 1 & Motor Leg Left & 1 & Motor Arm Right & 1 & & & & \\
\hline & & & & & Motor Leg Left & 1 & & & & & & & & \\
\hline & & & & & & & & & & & Motor Arm Right & 1 & Motor Arm Right & 1 \\
\hline 4 & Healthy (No Sym & nptoms) & & & & & & & & & Motor Leg Right & 1 & Motor Leg Right & 1 \\
\hline & & & & & & & Limb Ataxia & 1 & & & & & & \\
\hline 5 & Healthy (No Sym & nptoms) & & & & & & & & & & & & \\
\hline & & & & & & & & & & & & & & \\
\hline & & & Motor Leg Right & 1 & Motor Leg Left & 1 & & & & & & & & \\
\hline 6 & Healthy (No Sym & nptoms) & & & & & & & & & & & & \\
\hline & Visual & 2 & Visual & 2 & Visual & 2 & Facial Palsy & $N / A^{\star \star}$ & Facial Palsy & $N / A^{* *}$ & Visual & 2 & Visual & 2 \\
\hline 7 & & & Facial Palsy & 1 & Facial Palsy & 1 & Visual & 2 & Visual & 2 & & & & \\
\hline & & & & & & & & & & & & & & \\
\hline & & & & Part & icipant 4 & & & Part & ticipant 5 & & & Parti & icipant 6 & \\
\hline Pt & In-Person Neurc & ologist & Part 1 (Videc & & Part 21 & & Part 1 (Vide & & Part 2 & & Part 1 (Video & & \begin{tabular}{|l|} 
Part 2 (U \\
\end{tabular} & \\
\hline re. & Symptoms & NIHSS & Symptoms & NIHSS & Symptoms & NIHSS & Symptoms & NIHSS & Symptoms & NIHSS & Symptoms & NIHSS & Symptoms & NIHSS \\
\hline & Motor Arm Left & 1 & Visual & 1 & Visual & 1 & Limb Ataxia & 1 & Limb Ataxia & 1 & & & & \\
\hline 1 & Motor Leg Left & 1 & & & & & Visual & 1 & Visual & 1 & & & & \\
\hline & & & & & & & & & & & & & & \\
\hline & Facial Palsy & 1 & Dysarthria & 1 & Dysarthria & 1 & Dysarthria & 1 & Dysarthria & 1 & Dysarthria & 1 & Dysarthria & 1 \\
\hline & Motor Arm Left & 3 & Facial Palsy & 1 & Facial Palsy & 1 & Facial Palsy & 2 & Facial Palsy & 2 & Motor Arm Left & 3 & Motor Arm Left & 3 \\
\hline ? & Motor Leg Left & 1 & Motor Arm Left & 3 & Motor Arm Left & 3 & Motor Arm Left & 3 & Motor Arm Left & 3 & Motor Leg Left & $N / A^{* *}$ & Motor Leg Left & $N / A^{* *}$ \\
\hline 2 & & & Motor Leg Left & 2 & Motor Leg Left & 2 & Motor Leg Left & 1 & Motor Leg Left & 1 & & & & \\
\hline & & & & & & & & & & & & & & \\
\hline & \begin{tabular}{|l|} 
Motor Arm Left \\
\end{tabular} & 1 & LoC Questions & 1 & LoC Questions & 1 & Facial Palsy & 1 & Facial Palsy & 1 & Facial Palsy & 2 & Facial Palsy & 2 \\
\hline & Motor Leg Left & 1 & Motor Arm Left & 1 & Motor Arm Left & 1 & Limb Ataxia & 1 & Limb Ataxia & 1 & Motor Arm Left & 1 & Motor Arm Left & 1 \\
\hline 3 & & & Motor Leg Left & 1 & Motor Leg Left & 1 & LoC Questions & 1 & LoC Questions & 1 & Motor Leg Left & 1 & Motor Leg Left & 1 \\
\hline 3 & & & & & & & Motor Arm Left & 1 & Motor Arm Left & 1 & & & & \\
\hline & & & & & & & Motor Arm Right & 1 & Motor Arm Right & 1 & & & & \\
\hline & & & & & & & Motor Leg Left & 1 & Motor Leg Left & 1 & & & & \\
\hline & & & Dysarthria & 1 & Dysarthria & 1 & & & & & & & & \\
\hline 4 & Healthy (No Sym & pptoms) & Motor Leg Left & 1 & & & & & & & & & & \\
\hline & & & & & & & & & & & & & & \\
\hline & & & & & & & LoC Commands & $\mathrm{N} / \mathrm{A}^{* *}$ & LoC Commands & $N / A^{* *}$ & & & & \\
\hline 5 & Healthy (No Sym & iptoms) & & & & & & & & & & & & \\
\hline & & & & & & & & & & & & & & \\
\hline & & & Motor Leg Left & 1 & Motor Leg Left & 1 & LoC Commands & $\mathrm{N} / \mathrm{A}^{* *}$ & LoC Commands & $\mathrm{N} / \mathrm{A}^{* *}$ & Motor Leg Right & 1 & Motor Leg Left & 1 \\
\hline 6 & Healthy (No Sym & iptoms) & & & & & Motor Leg Left & 1 & Motor Leg Left & 1 & & & & \\
\hline & & & & & & & & & & & & & & \\
\hline & Visual & 2 & $\begin{array}{l}\text { Extinction and } \\
\text { Inattention }\end{array}$ & 1 & $\begin{array}{l}\text { Extinction and } \\
\text { Inattention }\end{array}$ & 1 & LoC Questions & $N / A^{* *}$ & LoC Questions & $N / A^{* *}$ & & & & \\
\hline 7 & & & Facial Palsy & 1 & Facial Palsy & 1 & Visual & 2 & Visual & 2 & & & & \\
\hline & & & Visual & 2 & Visual & 2 & & & & & & & & \\
\hline
\end{tabular}

${ }^{*} \mathrm{Pt}=$ Patient, Manipulated patients highlighted in blue

**Participant found symptom too difficult to score from the information provided

exhibited verbal or speech-related deficits that would necessitate listening to audio. Overall, 3 out of the 4 respondents indicated that the system was "generally accurate" and all 4 considered the interface to be "consistent".

For the respondents, the color-coded skeleton proved to be most helpful for their diagnoses while the joint movement graphs were not considered as important. The final survey revealed that the joint position graphs were superfluous for diagnosis; the quantitative position information was too granular to be useful to clinicians. Categorical representations of the data were preferred for future UI iterations. 
Table 4: Changes After Prototype UI Shown and Participant Belief in Accuracy of Presented Values*

\begin{tabular}{|c|c|c|c|c|c|c|c|c|c|c|c|c|c|c|c|c|c|c|}
\hline \multirow{2}{*}{ Pt. } & \multicolumn{6}{|c|}{ Difference in Confidence } & \multicolumn{6}{|c|}{ Changes Made? } & \multicolumn{6}{|c|}{ UI Incorrect? } \\
\hline & Part. 1 & Part. 2 & Part. 3 & Part. 4 & Part. 5 & Part. 6 & Part. 1 & Part. 2 & Part. 3 & Part. 4 & Part. 5 & Part. 6 & Part. 1 & Part. 2 & Part. 3 & Part. 4 & Part. 5 & Part. 6 \\
\hline 1 & 0 & -1 & 0 & 0 & -1 & 0 & No & Yes & No & No & No & No & Yes & No & Yes & Yes & Yes & Yes \\
\hline 2 & 0 & 1 & 0 & 0 & 0 & 1 & No & No & No & No & No & No & No & Yes & Yes & No & No & No \\
\hline 3 & 0 & 0 & 0 & 1 & 1 & 0 & Yes & No & No & No & No & No & Yes & Yes & No & No & No & Yes \\
\hline 4 & 0 & 0 & 0 & 0 & 0 & 0 & No & No & No & Yes & No & No & No & No & Yes & No & No & No \\
\hline 5 & 0 & 1 & 0 & 2 & -1 & 1 & No & Yes & No & No & No & No & No & No & No & No & No & No \\
\hline 6 & 0 & -1 & 0 & 1 & 0 & 0 & Yes & No & No & No & No & Yes & Yes & Yes & Yes & Yes & Yes & Yes \\
\hline 7 & 0 & 2 & 0 & 0 & 1 & 0 & No & No & No & No & No & No & Yes & No & No & Yes & No & No \\
\hline
\end{tabular}

${ }^{*} \mathbf{P t}=$ Patient, Part. = Participant, Manipulated patients highlighted

\section{DISCUSSION}

Our study uncovered a number of interesting results pertaining to: (1) the effects of computational aids on decision making and (2) whether clinicians were able to correct for errors made by the aids.

\subsection{Effects of Computational Aids on Clinical Decision Making}

An important concern raised by the participants of our focus group (as well as by the aforementioned study by Hirsch et al.) was the danger of relying too much on computational aids and how doing so would impact the diagnostic ability of clinicians [12]. Our results showed that computational aids did affect decision making, but only in specific instances. For example, as mentioned in Section 3.2, the UI helped Participant 1 align their assessment for Patient 3 closer to that of the in-person neurologist. After seeing the UI, Participant 1 included symptoms in their assessment that Patient 3 actually presented with (see Table 3). Despite this, Participant 1 did not use the absence of symptoms listed on the UI to remove symptoms they initially misidentified; symptoms like facial palsy and limb ataxia were not presented by Patient 3 . The UI therefore affected Participant 1's diagnosis for Patient 3 only partially, since Participant 1 did not remove symptoms after seeing the UI. Note also that Patient 3's information was manipulated (see Table 2). At first glance, it would seem that Participant 1 was affected by our manipulations since the UI swayed their assessment for Patient 3. However, Participant 1 noted that they suspected Patient 3's NIHSS score in the UI was too high, by looking at footage of the patient lifting her left leg against gravity; they were able to correctly identify our subtle manipulations.

Participant 2's changes for Patient 1 and Patient 5 were also of note. In the case of Patient 1, Participant 2 identified motor deficits only after seeing the UI, initially misdiagnosing the patient as healthy but later correcting themself. For Patient 5, Participant 2 did the opposite, changing their diagnosis to healthy (correct). Participant 2's decision making process was especially impacted by the data displayed in the UI. Their confidence decreased for Patient 1 after seeing the UI, and increased for Patient 5 when the UI helped them fix their initial, incorrect assessment. On the other hand, while Participant 4 made a negligible change in the NIHSS motor leg score of Patient 4, their overall assessment did not seem to be affected by the UI.

Participants 1 and Participant 4 seemed to be able to incorporate results shown in the UI into their decision making without letting the technological aid dominate their thinking, in contrast with the behavior of Participant 2. Clinicians seemed inclined to change their diagnoses only when the system definitively confirmed or denied their low confidence or "loosely held" beliefs, a conclusion supported by other work in the field of explainable AI [32]. Hirsch et al. arrived at a similar conclusion, showing that clinicians tended to trust a machine learning-based assessment system if the system's predictions conformed to their expectations (even if the system was incorrect) [12]. The behavior of Participant 3 further emphasizes this idea. Participant 3 was entirely unaffected by the UI, making no changes in the second part of the exam. Participant 3 reported perfect $7 / 7$ confidence in their own assessments in both parts of the experiment, a possible justification for why their decision making was not affected by the UI - Participant 3 had no loosely held beliefs that could have been affected by the UI. Refer to Table 4 .

It is unclear why Participant 5 was unaffected by the UI, especially given that they did not identify the manipulations for Patients 2 and 3 and did not have perfect confidence in their own assessments (Table 4). Further work needs to be done to understand why this individual was unaffected; perhaps issues with the design of the UI itself or the information presented may have discouraged Participant 5 from making use of the computational aid. (Participant 5 did not complete the final post-experiment survey.)

Note also that whether participants changed their responses in the second part of the exam did not relate to their role nor their experience level with the NIHSS. In fact, we did not notice any significant relationship between the roles (fellow, nurse practitioner, or neurologist) and how the UI affected decision making or their belief in the accuracy of results.

\subsection{Clinicians Correctly Catch Significant Errors in the UI}

Based on participants' belief in the accuracy of the results shown in the UI, large (erroneous) changes, like listing an otherwise unseen symptom for Patient 6, seemed to be easy to notice. Not all participants realized that the information for Patient 2 and Patient 3 were inaccurate; these manipulations were more subtle. Refer to Table 4. The ability to catch gross inaccuracies in the system would seem to indicate that respondents did not blindly accept the technological aid's results as true.

Hirsch et al. found that even when a machine-learning-based system was incorrect, clinicians accepted the accuracy of the displayed scores [12]. It is difficult, especially in healthcare, to build 
an AI-based system that is always correct [9]. We demonstrated on a small scale that clinicians were aware of possible computational mistakes and were able to identify them when they occur. Future work at the intersection of $\mathrm{AI}$ and human-computer interaction needs to focus on how to make these instances obvious; it should be made clear to clinicians that the results presented by computational aids are approximations and should be treated only as additional cues to incorporate in their clinical judgement. AI-based diagnostic aids need to add value to a decision, and cannot be expected to be a perfectly accurate diagnostic [33].

\subsection{Limitations and Future Work}

This study represents our early efforts to understand how stroke clinicians would be impacted by an AI-based computational aid. As such, the biggest limitation is the small participant pool of 6 . To be able to make more concrete, generalizable conclusions, a larger cohort of participants is required.

Diversity in the participant pool is also important. Unfortunately, we had a fairly homogeneous set of participants. Our manipulations may have had a more significant effect on those with less experience with the NIHSS. Hirsch et al. noted a difference in how novice and expert clinicians were affected by an automated assessment system; experts were better equipped to question the validity of the system's outputs, especially when they did not match their own subjective perceptions [12]. To check whether the same holds true for acute stroke diagnosis, a diverse cohort that includes non-stroke specialists like EMS personnel or less experience neurologists like medical residents is required. This work is ongoing.

To study concerns and information gaps in acute stroke diagnosis, we conducted a focus group comprised of 10 clinicians with a variety of roles, all of whom worked in the same clinic - the UCSD Stroke Center. Training relationships between seniors and juniors (between experienced neurologists and their fellows or residents, for example) and workplace hierarchies (like between nurses and physicians) may have affected the information that was shared in the focus group. Some may not have been comfortable sharing their concerns or areas of uncertainty in front of their coworkers, particularly the experienced, senior stroke specialists. To maximize the comfort of participants and to encourage them to freely share their concerns, we moderated the conversation to keep the focus on acute stroke diagnosis as a field, rather than on stroke diagnosis workflows at the UCSD Stroke Center in particular. Participant responses were therefore more generalizable and juniors did not have to bring up problems in their day-to-day jobs in front of their seniors. Despite our efforts, there may have still been discomfort that prevented individuals from sharing certain thoughts, affecting the conclusions we drew from the focus group. It would be beneficial to follow up individually with focus group participants to check one-on-one if they agreed with our conclusions. Moreover, we plan to conduct a focus group with a more heterogeneous set of stroke specialists, and not simply with those in the same healthcare center.

Transparency has been shown to engender trust in an automated system [15]. But the final survey revealed that when attempting to be transparent about how the computational aid arrived at predictions, it is possible to share too much. Survey respondents found that granular details that was not immediately readable or understandable - like the joint position graphs in Figure 1 - were superfluous in a time-sensitive, acute diagnosis setting. A balance has to be struck between presenting too much information for the sake of transparency and too little. We intend to explore alternative, more categorical representations of the rich spatial information captured by the Microsoft Kinect v2 in future UI iterations.

\section{CONCLUSION}

In this paper, we discussed our efforts to understand how practitioners would make use of a computational aid created to support acute stroke diagnosis. Our study uncovered a number of interesting results regarding the effects of a computational support system on decision making and whether clinicians were able to correct for errors made by the system. We showed that generally, clinicians balanced predictions from aids with their own assessments without letting the former dominate their thinking. Clinicians who participated could readily identify when the system was grossly incorrect and did not seem to blindly trust it. Lessons learned from this study provide guidance on properly designing computational aids for healthcare.

\section{REFERENCES}

[1] K. Agrawal, R. Raman, K. Ernstrom, R. J. Claycomb, D. M. Meyer, T. M. Hemmen, R. F. Modir, P. Kachhi, and B. C. Meyer. Accuracy of stroke diagnosis in telestroke-guided tissue plasminogen activator patients. Fournal of Stroke and Cerebrovascular Diseases, 25(12):2942-2946, 2016.

[2] D. Antipova, L. Eadie, A. Macaden, and P. Wilson. Diagnostic accuracy of clinical tools for assessment of acute stroke: a systematic review. BMC emergency medicine, 19(1):49, 2019.

[3] ASA. Why getting quick stroke treatment is important, 2018

[4] J. S. Ash, D. F. Sittig, E. G. Poon, K. Guappone, E. Campbell, and R. H. Dykstra. The extent and importance of unintended consequences related to computerized provider order entry. Journal of the American Medical Informatics Association, 14(4):415-423, 2007.

[5] S. Bacchi, T. Zerner, L. Oakden-Rayner, T. Kleinig, S. Patel, and J. Jannes. Deep learning in the prediction of ischaemic stroke thrombolysis functional outcomes: A pilot study. Academic radiology, 2019.

[6] J. C. Brigham. Target person distinctiveness and attractiveness as moderator variables in the confidence-accuracy relationship in eyewitness identifications. Basic and Applied Social Psychology, 11(1):101-115, 1990.

[7] E. M. Campbell, D. F. Sittig, K. P. Guappone, R. H. Dykstra, and J. S. Ash. Overdependence on technology: an unintended adverse consequence of computerized provider order entry. In AMIA Annual symposium proceedings, volume 2007, page 94. American Medical Informatics Association, 2007.

[8] O. Chernyshev, S. Martin-Schild, K. Albright, A. Barreto, V. Misra, I. Acosta, J. Grotta, and S. Savitz. Safety of tpa in stroke mimics and neuroimaging-negative cerebral ischemia. Neurology, 74(17):1340-1345, 2010.

[9] P. Croskerry. Clinical cognition and diagnostic error: applications of a dual process model of reasoning. 2, 14(1):27-35, 2009.

[10] A. Fouse, N. Weibel, E. Hutchins, and J. D. Hollan. Chronoviz: a system for supporting navigation of time-coded data. In CHI'11 Extended Abstracts on Human Factors in Computing Systems, pages 299-304. ACM, 2011.

[11] F. Gotoh, Y. Terayama, T. Amano, and S. S. C. of the Japan Stroke Society. Development of a novel, weighted, quantifiable stroke scale: Japan stroke scale. Stroke, 32(8):1800-1807, 2001.

[12] T. Hirsch, C. Soma, K. Merced, P. Kuo, A. Dembe, D. D. Caperton, D. C. Atkins, and Z. E. Imel. " it's hard to argue with a computer" investigating psychotherapists' attitudes towards automated evaluation. In Proceedings of the 2018 Designing Interactive Systems Conference, pages 559-571, 2018.

[13] C. Kambhampati, P. Drew, A. Ramesh, and J. Monson. Artificial intelligence in medicine. Annals of the Royal College of Surgeons of England, 86(5), 2004.

[14] C. E. Kimble and S. D. Seidel. Vocal signs of confidence. Fournal of Nonverbal Behavior, 15(2):99-105, 1991.

[15] J. B. Lyons, G. G. Sadler, K. Koltai, H. Battiste, N. T. Ho, L. C. Hoffmann, D. Smith, W. Johnson, and R. Shively. Shaping trust through transparent design: theoretical and experimental guidelines. In Advances in human factors in robots and unmanned systems, pages 127-136. Springer, 2017. 
[16] G. Mantokoudis, A. S. S. Tehrani, J. C. Kattah, K. Eibenberger, C. I. Guede, D. S Zee, and D. E. Newman-Toker. Quantifying the vestibulo-ocular reflex with video-oculography: nature and frequency of artifacts. Audiology and Neurotology, 20(1):39-50, 2015

[17] B. C. Meyer and P. D. Lyden. The modified national institutes of health stroke scale: its time has come. International journal of stroke, 4(4):267-273, 2009.

[18] M. A. Murphy, C. Willén, and K. S. Sunnerhagen. Kinematic variables quantifying upper-extremity performance after stroke during reaching and drinking from a glass. Neurorehabilitation and neural repair, 25(1):71-80, 2011.

[19] H. S. Nam, E. Park, and J. H. Heo. Facilitating stroke management using modern information technology. Fournal of stroke, 15(3):135, 2013

[20] N. I. of Health. Nih stroke scale. https://www.stroke.nih.gov/documents/NIH Stroke_Scale_508C.pdf.

[21] R. Parsons, D. Schembri, K. Hancock, A. Lonergan, C. Barton, T. Schermer A. Crockett, P. Frith, and T. Effing. Effects of the spirometry learning module on the knowledge, confidence, and experience of spirometry operators. NPf primary care respiratory medicine, 29(1):1-8, 2019.

[22] V. Rajan, S. Bhattacharya, R. Shetty, A. Sitaram, and G. Vivek. Clinical decision support for stroke using multi-view learning based models for nihss scores. In Pacific-Asia Conference on Knowledge Discovery and Data Mining, pages 190-199. Springer, 2016.

[23] V. Ramesh, K. Agrawal, B. Meyer, G. Cauwenberghs, and N. Weibel. Exploring stroke-associated hemiparesis assessment with support vector machines. In Proceedings of the 11th EAI International Conference on Pervasive Computing Technologies for Healthcare, pages 464-467. ACM, 2017.

[24] V. Ramesh, K. Agrawal, B. Meyer, G. Cauwenberghs, and N. Weibel. Strokeassociated hemiparesis detection using body joints and support vector machines. In Proceedings of the 12th EAI International Conference on Pervasive Computing Technologies for Healthcare, pages 55-58. ACM, 2018.

[25] V. Ramesh, S. Kim, H.-A. Nguyen, K. Agrawal, B. C. Meyer, and N. Weibel. Developing aids to assist acute stroke diagnosis, 2020.
[26] V. Ramesh, S. Rick, B. Meyer, G. Cauwenberghs, and N. Weibel. A Neurobehaviora Evaluation System Using 3D Depth Tracking \& Computer Vision: The Case of Stroke-Kinect. In Proceedings of Neuroscience 2016, Annual Meeting of the Society for Neuroscience (Poster presentation), San Diego, CA, USA, Nov. 2016.

[27] K. Robertson. Mindful use of health information technology. AMA fournal of Ethics, 13(3):193-196, 2011.

[28] L. H. Schwamm, E. S. Rosenthal, A. Hirshberg, P. W. Schaefer, E. A. Little, J. C. Kvedar, I. Petkovska, W. J. Koroshetz, and S. R. Levine. Virtual telestroke support for the emergency department evaluation of acute stroke. Academic Emergency Medicine, 11(11):1193-1197, 2004.

[29] M. Sendak, M. C. Elish, M. Gao, J. Futoma, W. Ratliff, M. Nichols, A. Bedoya, S. Balu, and C. O'Brien. " the human body is a black box" supporting clinical decision-making with deep learning. In Proceedings of the 2020 Conference on Fairness, Accountability, and Transparency, pages 99-109, 2020.

[30] L. L. W. Sim, K. H. K. Ban, T. W. Tan, S. K. Sethi, and T. P. Loh. Development of a clinical decision support system for diabetes care: A pilot study. PloS one, 12(2): $\mathrm{e} 0173021,2017$.

[31] P. Vilela. Acute stroke differential diagnosis: stroke mimics. European journal of radiology, 96:133-144, 2017.

[32] D. Wang, Q. Yang, A. Abdul, and B. Y. Lim. Designing theory-driven user-centric explainable ai. In Proceedings of the 2019 CHI Conference on Human Factors in Computing Systems, pages 1-15, 2019.

[33] Q. Yang, A. Steinfeld, and J. Zimmerman. Unremarkable ai: Fitting intelligent decision support into critical, clinical decision-making processes. In Proceedings of the 2019 CHI Conference on Human Factors in Computing Systems, pages 1-11, 2019.

[34] K. S. Yew and E. Cheng. Acute stroke diagnosis. American family physician, 80(1):33, 2009.

[35] J. Yu, D. Kim, H. Park, S.-c. Chon, K. H. Cho, S.-J. Kim, S. Yu, S. Park, and S. Hong. Semantic analysis of nih stroke scale using machine learning techniques. In 2019 International Conference on Platform Technology and Service (PlatCon), pages 1-5. IEEE, 2019. 